\title{
Effectiveness of Progressive Muscle Relaxation in Reducing Academic Stress of Secondary Schools Students of Kerala
}

\author{
Praseeda P. Nair ${ }^{1}$, Dr. K.P. Meera ${ }^{2}$ \\ ${ }^{I}$ (Senior Research Fellow, Department of Education, University of Calicut, Kerala). \\ ${ }^{2}$ (Associate Professor, Department of Education, University of Calicut, Kerala).
}

\begin{abstract}
Students are in such a competitive busy world that they have to struggle with lot of stressors in their daily life. This may affect their achievement in schools and future life and also lead to psychosomatic illness. So the present study focused on the importance and effectiveness of giving progressive muscle relaxation for students to reduce their academic stress. The result of the study revealed that Progressive Muscle Relaxation is significantly effective for reducing academic stress of students.
\end{abstract}

Key words: Academic stress, Progressive Muscle Relaxation (PMR).

\section{Introduction}

Students have to struggle with a lot of stressors in their day to day life. One of the major stress they have to face is academic stress. Academic stress is a combination of various other stressors. It was found that various stressors can lead to various psychosomatic illness. This may affect the students adversely in their life. So there is need for different measures to reduce stress of students. There are many intervention programmes for reducing students' stress. Various studies have been conducted to investigate the role of relaxation techniques in reducing stress and stress related illness. Relaxation training is probably the most used pharmacological, both stand-alone and psychotherapy combined approach for the treatment for any medical and psychological diseases. Among the wide range of many non-conventional and doubtful treatments, relaxation based methods such as meditation, progressive muscle relaxation, mindfulness and autogenic training had received great scientific attention and validation (Pagneni, Manzoni, Gian, Castelunovo and Molinari, 2009) [1]. Relaxation techniques belong to the main coping strategies that are supplied to children to improve Stress Management (Lohaus and Kleen-hebling, 2000) [2]. Relaxation training is effective in reducing anxiety in any kind of participants, male or female, young or old, affective or not by physical or psychological disorders (Pagnini, et al., 2009). Dave (2009) [3] recommended to teach students relaxation techniques to help them feel calm. Lohaus and Kleim-hebling (2000) concluded in their study that children who participated in relaxation activities during school, experience reduced blood pressure, and heart rate and their self report ensured improvement in feelings of calmness and psychological well being.

Lapota, Nida, Marable (2006) [4] opined that progressive muscle relaxation technique reduce arousal, improve behavioural self-regulation and prevent physical aggression among students with EBD. Relaxation techniques significantly reduce test anxiety and improve working memory capacity and its components storage and processing and Academic Achievement (Larson, El Ramahi, Conn, Ester \& Ghibellini, 2010) [5].

According to Bruno-Golden (1987) [6] the efforts of relaxing carried with it autonomic responses which are opposite and antagonistic to these characteristics of anxiety and other symptom-related stresses and relaxation is also a means to develop a coping skill. Preventive stress inoculation program for adolescents consisting of progressive muscle relaxation along with cognitive restricting and assertiveness training reduced anxiety and stress related symptom of post test (Kiselica, Baker, Thomas \& Reedy, 1994) [7]. Reviewing various investigations the researchers sought to ascertain the effectiveness of Progressive Muscle Relaxation training in reducing the academic stress of secondary school students.

\section{Progressive Muscle Relaxation (PMR)}

Wolpe's initial modification of Jacobson's Original Progressive Relaxation Training became a standard tool among therapist of all types, especially those offering behavioural and cognitive-behavioural therapy. Through clinical and empirical evaluations its value as a component of a variety of treatment programs has been well established (Bernstein, Borkovec \& Hazelett-Stevens, 2000) [8]. Jacobson (1976) [9] explained that relaxation in any muscle means complete absence of all contractions. Jacobson discovered that systematic tensing and releasing dozens of muscle groups, learning to attend and discriminate between resulting sensation of tension and relaxation can help a person to completely eliminate muscle contractions and experience a feeling of deep relaxation. Jacobson suggested sixteen muscle groups for the sequential contraction and relaxation which will lead to complete deep relaxation. 


\section{Statement Of The Problem}

Effectiveness of Progressive Muscle Relaxation in Reducing Academic Stress of Secondary Schools Students of Kerala

\section{Variables Of The Study}

The present study involved following variables

\subsection{Independent Variable}

For the present study Progressive Muscle Relaxation Training was selected as independent variable.

\subsection{Dependent Variable}

Academic Stress was taken as dependent variable.

\section{Research Question}

1. To find out whether there exist any difference in academic stress before and after progressive muscle relaxation exercise for the total sample.

2. To find out whether there exist any difference in academic stress before and after the PMR exercise for boys.

3. To find out whether there exist any difference in academic stress before and after PMR exercise for girls.

4. To find out whether there exist any difference in pretest of academic stress based on gender.

5. To find out whether there exist any difference in post test of academic stress based on gender.

\section{Hypotheses Of The Study}

1. There exists significant difference in the mean pre test and post test scores of academic stress for the total sample.

2. There exists significant difference between the mean pre test and post test scores of academic stress for subsample boys.

3. There exists significant difference between the mean pre test and post test scores of academic stress for subsample girls.

4. There exists no significant difference in the mean pretest scores of academic stress based on gender.

5. There exists significant difference in the mean post test scores of academic stress based on gender.

\subsection{Design of the Study}

\section{Methodology}

Quasi experimental study with single group pre test - post test design.

\subsection{Instruments Used}

\subsubsection{Academic Stress Inventory (Praseeda \& Meera, 2012)}

Academic Stress Inventory developed by the investigators was a likert type scale with 52 items. Inventory was standardized with reliability of .81 and criterion validity .87 . Content validity and face validity of the inventory was also established.

\subsubsection{Progressive Muscle Relaxation Training (Adapted for of Jacobson's Progressive Muscle Relaxation) 6.3 Sample}

The sample of the study consisted of 60 secondary school students giving 1:1 proportion for gender. The sample was collected from Kerala.

\subsection{Procedure}

Academic stress inventory was administered on the sample before giving the Progressive Muscle Relaxation to obtain pretest scores. The PMR exercise was given to the students for a period of 6 weeks. Exercise was given for five days a week for 30 minutes. After completing the exercise after 6 weeks Academic Stress Inventory was again administered to get the post test scores. The data obtained is then analyzed.

\section{Statistical Techniques Used}

Test of significance of difference between mean was used to compare the mean pre test, post test scores of total sample and the mean pre test post scores based on gender.

Test of significance of difference between mean was used to compare the mean pre-test scores of Boys and Girls and the mean post test scores of Boys and Girls. 


\subsection{Result 1}

\section{Results And Discussions}

The effect of Progressive Muscle Relaxation for reducing academic stress of secondary school students was found out by computing the ' $t$ ' using paired t-test. It was then tested for significance. The consolidated result of mean difference analysis of pre test and post test scores of total sample are given in Table 1.

TABLE 1 Result of Test of Significance of Difference between Mean Pre test and Post test Scores on Academic Stress of Secondary School Students for Total Sample

\begin{tabular}{llllll}
\hline \multicolumn{1}{c}{ Groups } & $\mathrm{N}$ & Mean & SD & $\mathrm{t}$ & Level of Significance \\
\hline Pre test & 60 & 168.85 & 43.845 & \multirow{2}{*}{6.768} & \multirow{2}{*}{01} \\
Post test & 60 & 137.92 & 40.909 & \\
\hline
\end{tabular}

From table 1, it is found that the t-value obtained is 6.768 which is greater than the table value 2.58 required for significant at 0.01 level. Hence it is clear that there exist significant difference between mean pre test and post test scores on Academic Stress of secondary school students. The post test mean score is significantly lower than pre-test score which clearly proves that Progressive Muscle Relaxation is effective in reducing academic stress in classroom situation for the total sample.

\subsection{Result 2}

The consolidated results of the test of significance of difference between mean pretest and mean posttest scores on Academic Stress of boys before and after PMR exercise is given in Table 2

TABLE 2 Result of Test of Significance of Difference between Mean Pre test and Post test Scores on Academic Stress of Secondary School Students for the Sample Boys

\begin{tabular}{ccccccc}
\hline Groups & N & Mean & SD & df & t & Level of Significance \\
\hline Pre test & 30 & 159.53 & 36355 & 29 & \multirow{2}{*}{4.84} & 0.01 \\
Post test & 30 & 126.47 & 34.99 & & & \\
\hline
\end{tabular}

Table 2 shows that the t-value obtained are 4.84 which is greater than the table value required for significant at 0.01 level. Hence it is clear that there exist significant difference between mean pre test and post test scores on Academic Stress of secondary school students-subsample Boys. The post test mean score is significantly lower than pre-test score which clearly proves that Progressive Muscle Relaxation is effective in reducing academic stress in classroom situation for the subsample Boys.

\subsection{Result 3}

The consolidated results of the test of significance of difference between mean pretest and mean posttest scores on Academic Stress of Girls before and after PMR exercise is given in Table 2.

TABLE 3 Result of Test of Significance of Difference between Mean Pre test and Post test Scores on Academic Stress of Secondary School Students for the Sample Girls

\begin{tabular}{|c|c|c|c|c|c|c|}
\hline Groups & $\mathrm{N}$ & Mean & SD & $\mathrm{t}$ & $\mathrm{df}$ & $\begin{array}{c}\text { Level of } \\
\text { Significance }\end{array}$ \\
\hline Pre & 30 & 178.17 & 48.94 & \multirow{2}{*}{4.679} & \multirow{2}{*}{29} & \multirow{2}{*}{0.01} \\
\hline Post & 30 & 149.37 & 43.69 & & & \\
\hline
\end{tabular}

Table 3 shows that the t-value obtained are 4.679 which is greater than the table value required for significance at 0.01 level. Hence it is clear that there exist significant difference between mean pre test and post test scores on Academic Stress of secondary school students-subsample Girls. The post test mean score is significantly lower than pre-test score which clearly proves that Progressive Muscle Relaxation is effective in reducing academic stress in classroom situation for the subsample Girls.

\subsection{Result 4}

The consolidated results of the test of significance of difference between mean pretest scores on Academic Stress based on gender of secondary school students. Data and result of the t-test are presented in Table 4.

TABLE 4MResult of Test of Significance of Difference between Mean Pre test Scores on Academic Stress of Secondary School Students Based on Gender

\begin{tabular}{cccccccc}
\hline & Groups & $\mathrm{N}$ & Mean & SD & t & df & Significance \\
\hline \multirow{2}{*}{ Pre test } & Boys & 30 & 159.53 & 36.551 & \multirow{2}{*}{1.671} & \multirow{2}{*}{ Not Significant } \\
& Girls & 30 & 178.17 & 48.940 & & \\
\hline
\end{tabular}


Table 4 shows that the obtained t-value is less than the table value 1.96 at 0.05 level of significance. Hence it is clear that there is no significant difference between the means of pretest scores of Academic Stress based on gender. This implies that there is no significant difference in the pre experimental status of secondary school students based on gender.

\subsection{Result 5}

The consolidated results of the test of significance of difference between mean post test scores on Academic Stress based on gender of secondary school students. Data and result of the t-test are presented in Table 5 .

TABLE 5 Result of Test of Significance of Difference between

Mean Post test Scores on Academic Stress of Secondary School Students Based on Gender

\begin{tabular}{cccccccc}
\hline \multicolumn{2}{c}{ Groups } & N & Mean & SD & t & df & $\begin{array}{c}\text { Level of } \\
\text { Significance }\end{array}$ \\
\hline \multirow{2}{*}{ Post test } & Boys & 30 & 126.47 & 34.98 & 2.241 & \multirow{2}{*}{58} & 0.05 \\
& Girls & 30 & 149.37 & 43.69 & & \\
\hline
\end{tabular}

Table 5 shows that the obtained t-value is greater than the table value 1.96 at .05 level of significance. This clear that there exist significant difference between the means posttest scores of Academic Stress based on gender. This implies that Boys and Girls differ significantly in their mean post test scores. The mean post test scores of boys is significantly lower than that of girls which shows that Progressive Muscle Relaxation is more effect in reducing Academic Stress of Boys.

\section{Conclusion}

The present study explored the effectiveness of Progressive Muscle Relaxation as an intervention to reduce the academic stress of students. Investigators studied whether Progressive Muscle Relaxation can bring down the Academic Stress of students. The effectiveness of Progressive Muscle Relaxation was measured by comparing the pre test and post test scores of the sample. The result indicated that Progressive Muscle Relaxation is an effective intervention in reducing the Academic Stress of secondary school students in the classroom situation. As the reduced rate of Stress is essential for the well being school should take necessary actions to include such relaxation exercises in school, as such techniques are useful for controlling their stress it is useful for them for their future also. Special time can be allotted for such intervention or it can be either integrated with physical education. Only secondary schools students were considered for the present study. The study can be conducted on different sample like primary, higher secondary, higher education, etc. Stress Reduction Programmes can also be extended to teachers for their better performance and wellbeing.

\section{References}

[1] Pagnini, F., Gian, M.M., Castelnuovo, G., \& Molinari, E. (2009). Efficacy of relaxation training in treating anxiety. International Journal of Behavioural Consultation and Therapy, 5(3-4), 264-269.

[2] Lohaus, A., \& Klein-hebling, J. (2009). Coping in childhood: A comparative evaluation of different relaxation technique. Anxiety, Stress and Coping: An international Journal, 13(2), 187-211.

[3] Dave, V. (2009). Meeting the needs, disaffected students: Engaging students with social, emotional and behavioural difficulties. London, New York: Continuum Education.

[4] Lapota, C., Nida, R.E., \& Marable, M.A. (2006). Progressive muscle relaxation: Preventing aggression in students with EBD. Teaching Exceptional Children, 38(4), 20-25.

[5] Larson, B., \& Carlson, J. (1995). A school-based, nurse-administered relaxation training for children with chronic tension- Type headache. Journal of Paedatric Psychology, 21(5), 603-614.

[6] Bruno-Golden, B. (1987). Theoretical considerations and Applications of relaxation training for children. A Journal of the Royal Society for the Promotion of Health, 17(2), 51-54.

[7] Kiselica, M.S., Baker, S.B., Thomas, R.N., \& Reddy, S. (1994). Effect of stress inoculation training on anxiety. Stress and Academic Performance Among Adolescents, 41(3), 335-42.

[8] Jacobson. (1976). You must relax. London: Unwin Paperbacks.

[9] Bernstein, D.A., Borkovec, J.D., \& Hazlettsevens. (2000). New directions in progressive relaxation training: A guidebook for helping professionals. London: Prager. 